\title{
QUADRATIC INTEGRALS OF MOTION AND STELLAR ORBITS IN THE ABSENCE OF AXIAL SYMMETRY OF THE POTENTIAL
}

\author{
G.G. Kuzmin \\ Tartu Astrophysical Observatory \\ 202444 Toeravere, Estonia, USSR
}

As is well known, in the case of an axially symmetric and time-invariant gravitational potential, if the potential satisfies one particular additional constraint, there exist three isolating integrals of motion: the energy integral, the area integral, and the third integral which is quadratic in the velocities. This work discusses the case in which there exist quadratic integrals in the absence of axial symmetry of the potential. Such a case has already been examined by Eddington [1], but in their explicit form, the integrals were introduced by Clark [2].

Let us use, following Eddington and Clark, an ellipsoidal coordinate system $q_{1}, q_{2}, q_{3}$. The coordinate surfaces (which are orthogonal) represent a family of second-order confocal surfaces. Let this coordinate system be co-axial with Cartesian coordinates $x, y, z$. Moreover, let $z$ be the axis with which all the coordinate surfaces (hyperboloids as well as ellipsoids) have a real intersection. Then, on the $z$-axis, either $z=q_{1}$ (hyperboloids of two sheets), or $|z|=q_{2}$ (hyperboloids of one sheet), or $|z|=q_{3}$ (ellipsoids). The domains of the $q_{i}$ are $-\alpha \leq q_{1} \leq \alpha, \alpha \leq q_{2} \leq \beta$, $\beta \leq q_{3}$, where $\alpha$ and $\beta$ are parameters of the coordinate system (determined by the intersection of the focal curves with the $z$-axis).

Let $v_{i}$ be the velocities and $p_{i}$ the generalized (specific) momenta along the coordinate line $q_{i}$. In the ellipsoidal coordinate system:

$$
\frac{d q_{i}}{d t}=\sqrt{g_{i}} v_{i}=g_{i} p_{i}, \quad(i=1,2,3)
$$

( $t$ is the time), while

$$
g_{i}=\frac{\left(q_{i}^{2}-\alpha^{2}\right)\left(q_{i}^{2}-\beta^{2}\right)}{\left(q_{i}^{2}-q_{k}^{2}\right)\left(q_{i}^{2}-q_{l}^{2}\right)}, \quad(k, l \neq i) .
$$

Obviously, $0 \leq g_{i} \leq 1$, and as can easily be shown, $\sum g_{i}=1$. On the $z$-axis, $g_{i}=1$ in its "own" domain, i.e. where $z$ (or $|z|$ ) equals $q_{i}$, and $g_{i}=0$ outside this domain.

From: The Dynamics of Galaxies and Star Clusters, Proceedings of a National Conference in Alma-Ata, October 23-26, 1972, p. 71-75, ed. Omarov, T.B. ("Nauka" of the Kazakh S.S.R., Alma-Ata, 1973). Translated from the Russian by Alex Shprintsen at CITA, Toronto. 
Clark $[2]$, using Eddington's work, presented differential equations which the potential $\Phi$ must satisfy if the three isolating quadratic integrals are to exist. However, he did not find solutions to these equations. The solution has the following simple form:

$$
\Phi=\sum_{i} g_{i} \phi_{i}, \quad \phi_{i}=\phi\left(q_{i}\right)
$$

Formally, the solution contains three arbitrary functions $\phi_{i}$. But they should be viewed as parts of one single function $\phi(q)$ where $q \geq-\alpha$. On the $z$-axis, it is obvious that $\Phi=\phi(z)$, or $\phi(|z|)$ when $|z| \geq \alpha$.

The three independent quadratic isolating integrals of motion have the following form:

$$
I_{j}=\sum_{i} h_{i j}\left(\frac{1}{2} p_{i}^{2}-\phi_{i}\right), \quad(j=1,2,3)
$$

where

$$
\begin{aligned}
h_{i j} & =a_{i j} g_{i}, \\
a_{i 1}=1, \quad a_{i 2} & =\frac{q_{k}^{2}+q_{l}^{2}}{\alpha^{2}+\beta^{2}}, \quad a_{i 3}=\frac{q_{k}^{2} q_{l}^{2}}{\alpha^{2} \beta^{2}} .
\end{aligned}
$$

The properties of the coefficients $h_{i j}$ are analogous to the properties of the coefficients $g_{i}$ as presented above. Obviously, $I_{1}$ is the energy integral.

Using Poisson's equation for the density $\rho$, we derive the expression:

$$
\rho=\sum_{i, k} g_{i} g_{k} \rho_{i k}
$$

where

$$
4 \pi G \rho_{i i}=\Psi_{i}=\Psi\left(q_{i}\right), \quad 4 \pi G \rho_{i k}=\frac{2 \int_{q_{i}}^{q_{k}} \Psi(q) q d q}{q_{k}^{2}-q_{i}^{2}} .
$$

Here $G$ is the gravitational constant, and $\Psi(q)$ is an arbitrary function linked to the arbitrary function $\phi(q)$ by the equation:

$$
\phi^{\prime \prime}+\left(\frac{1}{q^{2}-\alpha^{2}}+\ldots\right) q \phi^{\prime}-\left[\frac{2 \alpha^{2}}{\left(q^{2}-\alpha^{2}\right)^{2}}\left(\phi-\phi_{\alpha}\right)+\ldots\right]+\Psi=0
$$

where the ellipses stand for a similar term for $\beta$, and $\phi_{\alpha}=\phi(\alpha)$. Obviously, on the $z$-axis, $4 \pi G \rho=\Psi(z)$ (or $\Psi(|z|)$ when $|z| \geq \alpha)$.

The expression for the density (6) (as well as the expression for the potential (3)) is a generalization of the expression derived for axial symmetry [3]. Theorems for the non-negativity of the density and the finiteness of the mass remain valid: the density is everywhere non-negative if it is non-negative on the $z$-axis; the mass is finite if $\Psi q^{3} \rightarrow 0$ when $q \rightarrow \infty$ (both conditions are necessary and sufficient). The theorem concerning the equatorial plane of symmetry also remains valid [4]. The potential and the density are obviously symmetric relative to the planes $x=0$ and $y=0$. But if the stellar system is self-gravitating (so that $\Psi=\Psi\left[\phi(q), q^{2}\right]$ ), then it is also symmetric to the plane $z=0(\phi(-q)=\phi(q)$ when $-\alpha \leq q \leq \alpha)$.

Having set up the function $\Psi(q)$ (or $\phi(q)$ ), we derive a model for the mass distribution, allowing for the integral of motion (4). If $\Psi(q)$ decreases rapidly 
with $|q|$, then the models are greatly flattened along $z$. Such models are treated similarly to the greatly flattened, axisymmetric models $[3 \mid$. Ellipsoidal models are also possible. They may be derived by taking:

$$
\Psi(q)=4 \pi G \rho_{0}\left(1+\frac{q^{2}}{\gamma^{2}}\right)^{-2} .
$$

Then

$$
\rho=\rho_{0}\left(1+\frac{x^{2}}{\beta^{2}+\gamma^{2}}+\frac{y^{2}}{\alpha^{2}+\gamma^{2}}+\frac{z^{2}}{\gamma^{2}}\right)^{-2}
$$

( $\rho_{0}$ is the central density, $\gamma$ is a parameter). This is the generalization of the axisymmetric ellipsoidal models examined earlier [3].

Let us now turn to the problem of stellar orbits. From expression (4) for the integrals of motion $I_{j}$, we find:

$$
\frac{p_{i}^{2}}{2}=\frac{Q_{i}}{\left(q_{i}^{2}-\alpha^{2}\right)\left(q_{i}^{2}-\beta^{2}\right)}+\phi_{i}, \quad Q_{i}=Q\left(q_{i}\right),
$$

where

$$
Q(q)=I_{1} q^{4}-\left(\alpha^{2}+\beta^{2}\right) I_{2} q^{2}+\alpha^{2} \beta^{2} I_{3} .
$$

Using $p_{i}$, it is possible to express the remaining three independent integrals of motion:

$$
I_{j+3}=\sum_{i} \int \frac{\partial p_{i}}{\partial I_{j}} d q_{i}+ \begin{cases}-t, & j=1, \\ 0, & j=2,3 .\end{cases}
$$

The first of these integrals is non-conservative, the other two are conservative but non-isolating. With fixed $I_{1}, I_{2}, I_{3}$, the expressions for the integrals (13) become the equations of the orbit.

The orbits are bounded by the surfaces $q=$ const. We derive the values of $q$ by solving the equation

$$
Q(q)+\left(q^{2}-\alpha^{2}\right)\left(q^{2}-\beta^{2}\right) \phi(q)=0 .
$$

Three solutions for $q^{2}$ correspond to a finite orbit (assuming there is equatorial symmetry). The negativity of the third derivative of the second term with respect to $q^{2}$ is the condition for there to be no more than 3 solutions. There exist four classes of orbits: 1) box orbits, 2) horizontal annular, 3) external vertical annular, and 4) internal vertical annular orbits. The three solutions for $q^{2}$ are to be found respectively in the domains: $q_{1}, q_{2}, q_{3} ; q_{1}, q_{3}(2) ; q_{2}, q_{3}(2) ; q_{2}(2), q_{3}$.

Box orbits are reversible: after entering the corner of the box, the direction of stellar motion is reversed. Annular orbits are irreversible: motion is possible in two opposite directions, but a reversal of direction is not possible. The phase density of stars, which in a stationary state is a function of the isolating integrals $I_{j}$, may depend on them in the domain of irreversible orbits in a double-valued fashion. This makes possible centroid motion, despite the quadratic nature of the integrals.

On the boundaries between the four indicated classes, orbits are unstable, and there exist asymptotic orbits. 


\section{REFERENCES}

1. Eddington, A.S., 1916. Mon. Not. R. astr. Soc., 76, 37.

2. Clark, G.L., 1936. Mon. Not. R. astr. Soc., 97, 182.

3. Kuzmin, G.G., 1956. Astr. Zh., 33, 27. (Tartu Astr. Obs. Teated, 2).

4. Kuzmin, G.G., 1963. Publ. Tartu Astroph. Obs., 34, 18. 